\title{
Esophageal Perforation Accompanying Mediastinitis in Blunt Trauma in a Patient with Thoracic Osteophytes
}

\author{
Kei Suzuki ${ }^{1-3}$, Haruna Yoshida ${ }^{1}$, Ryo Esumi ${ }^{1}$, Yohei Ieki ${ }^{1}$, Akira Yamamoto ${ }^{4}$, Masaki Ohi ${ }^{4}$, \\ Tadashi Kaneko ${ }^{1}$ and Hiroshi Imai ${ }^{1}$
}

\begin{abstract}
:
We herein report a 61-year-old man who sustained injury after a 2-m fall and developed mediastinitis. He presented to another hospital two days after the fall and was transferred to our hospital four days after the fall with a fever and dysphagia. Computed tomography revealed osteophytes on the second and third thoracic vertebrae and free air in the mediastinum, indicating esophageal perforation. Emergent surgery was performed. Intraoperatively, a longitudinal esophageal tear was identified. We stress the importance of being aware of the possibility of osteophyte-related esophageal perforation in patients with a history of a fall. A delayed diagnosis affects the prognosis.
\end{abstract}

Key words: Esophageal perforation, thoracic osteophytes, blunt trauma, multidisciplinary approach

(Intern Med Advance Publication)

(DOI: 10.2169/internalmedicine.8930-21)

\section{Introduction}

Esophageal perforations are a relatively rare but difficult issue to manage with a high mortality rate, especially in cases with a delayed diagnosis $(1,2)$. Esophageal perforations may be spontaneous, iatrogenic, or malignant (1).

We herein report the first case of mediastinitis from esophageal perforation with septic shock that required subtotal esophagectomy. The perforation resulted from blunt trauma that caused hyperextension of the upper thoracic spine, which had developed osteophytes. The relationship between the spinal osteophytes, esophageal perforation, and closed injury after a fall is discussed.

\section{Case Report}

A 61-year-old Japanese man with a history of spinal spondylosis was brought to our hospital because of a fever and pneumomediastinum that had been detected on thoracic computed tomography (CT) performed at another hospital.

While working, he had landed heavily on his back after falling from a two-meter-high stepladder four days previously. He avoided visiting a hospital and observed whether his pain resolved; however, head and back pain persisted, and he visited a hospital 2 days previously. At that time, he had been diagnosed with a simple contusion because of negative findings on head CT. Because he was in a stable condition, no other studies besides head CT were performed. However, the back pain persisted, and he took time off from his job. He noticed dysphagia with a fever after visiting the previous hospital but continued to ingest his normal diet. However, his fever persisted, and he returned to the hospital complaining of swelling of the anterior cervical region and progressive dysphagia.

Upon transfer to our hospital, he was alert but presented with hypotension, tachycardia, and increased respiratory effort. His oxygen saturation was $99 \%$ under $2 \mathrm{~L} / \mathrm{min}$ oxygen administration with a respiratory rate of 34 breaths/min. His initial body temperature, pulse rate, and blood pressure were $38.1{ }^{\circ} \mathrm{C}, 110$ beats/min, and $97 / 55 \mathrm{mmHg}$, respectively. $\mathrm{He}$ complained of hoarseness with stridor and difficulty opening his mouth. A laboratory examination showed acute kidney injury, mainly owing to dehydration, and mild thrombocy-

\footnotetext{
${ }^{1}$ Emergency and Critical Care Center, Mie University Hospital, Japan, ${ }^{2}$ Department of Infectious Diseases, Mie University Hospital, Japan, ${ }^{3}$ Department of Hematology and Oncology, Mie University Graduate School of Medicine, Japan and ${ }^{4}$ Department of Gastrointestinal and Pediatric Surgery, Mie University Hospital, Japan

Received: October 29, 2021; Accepted: December 26, 2021; Advance Publication by J-STAGE: February 8, 2022

Correspondence to Dr. Kei Suzuki, keis@clin.medic.mie-u.ac.jp
} 
Table. Laboratory Findings at Presentation.

\begin{tabular}{cclclc}
\hline Hematology & \multicolumn{3}{c}{ Biochemistry } & \multicolumn{3}{c}{ Blood gas } \\
WBC & $5,070 / \mu \mathrm{L}$ & $\mathrm{TP}$ & $5.8 \mathrm{~g} / \mathrm{dL}$ & $\mathrm{pH}$ & $7.424 \mathrm{mmHg}$ \\
Neu & $4,715 / \mu \mathrm{L}$ & $\mathrm{Alb}$ & $2.6 \mathrm{~g} / \mathrm{dL}$ & $\mathrm{PaCO}_{2}$ & $34.5 \mathrm{mmHg}$ \\
$\mathrm{Ly}$ & $250 / \mu \mathrm{L}$ & $\mathrm{BUN}$ & $48.3 \mathrm{mg} / \mathrm{dL}$ & $\mathrm{PaO}_{2}$ & $138.8 \mathrm{mmol} / \mathrm{L}$ \\
Mo & $105 / \mu \mathrm{L}$ & $\mathrm{Cr}$ & $2.62 \mathrm{mg} / \mathrm{dL}$ & $\mathrm{HCO}_{3}-$ & $22.1 \mathrm{mmol} / \mathrm{L}$ \\
$\mathrm{RBC}$ & $334 \times 10^{4} / \mu \mathrm{L}$ & $\mathrm{Na}$ & $133 \mathrm{mEq} / \mathrm{L}$ & $\mathrm{BE}$ & $-2.3 \mathrm{mmol} / \mathrm{L}$ \\
$\mathrm{MCV}$ & $99.7 \mathrm{fL}$ & $\mathrm{K}$ & $4.0 \mathrm{mEq} / \mathrm{L}$ & Lactate & 2.2 \\
$\mathrm{Hb}$ & $11.6 \mathrm{~g} / \mathrm{dL}$ & $\mathrm{Cl}$ & $97 \mathrm{mEq} / \mathrm{L}$ & & \\
$\mathrm{Ht}$ & $33.3 \%$ & $\mathrm{Ca}$ & $8.4 \mathrm{mg} / \mathrm{dL}$ & Urinalysis & \\
Plt & $11.2 \times 10^{4} / \mu \mathrm{L}$ & $\mathrm{P}$ & $4.3 \mathrm{mg} / \mathrm{dL}$ & Gravity & 1.005 \\
& & $\mathrm{Mg}$ & $1.7 \mathrm{mg} / \mathrm{dL}$ & $\mathrm{pH}$ & 5.0 \\
Coagulation & & $\mathrm{AST}$ & $79 \mathrm{IU} / \mathrm{L}$ & WBC & $(-) \mathrm{mg} / \mathrm{dL}$ \\
APTT & $38.6 \mathrm{~s}$ & $\mathrm{ALT}$ & $51 \mathrm{IU} / \mathrm{L}$ & Protein & $30 \mathrm{mg} / \mathrm{dL}$ \\
PT & $14.1 \mathrm{~s}$ & $\mathrm{LDH}$ & $519 \mathrm{IU} / \mathrm{L}$ & Glucose & $(-)$ \\
PT-\% & $71.2 \%$ & ALP & $104 \mathrm{IU} / \mathrm{L}$ & Ketones & $(-)$ \\
PT-INR & 1.22 & T-Bil & $1.6 \mathrm{mg} / \mathrm{dL}$ & Blood & $(2+)$ \\
Fibrinogen & $>800 \mathrm{mg} / \mathrm{dL}$ & Glucose & $112 \mathrm{mg} / \mathrm{dL}$ & & \\
D-dimer & $3.96 \mu \mathrm{m} / \mathrm{mL}$ & $\mathrm{CPK}$ & $1413 \mathrm{IU} / \mathrm{L}$ & & \\
& & AMY & $25 \mathrm{IU} / \mathrm{L}$ & & \\
& & CRP & $40.40 \mathrm{mg} / \mathrm{dL}$ & & \\
\hline
\end{tabular}

WBC: white blood cell, Neu: neutrophil, Ly: lymphocyte, Mo: monocyte, RBC: red blood cells MCV: mean corpuscular volume, Hb: hemoglobin, Ht: hematocrit, Plt: platelet, APTT: activated partial thromboplastin time, PT: prothrombin time, PT-INR: prothrombin-international normalized ratio, TP: total protein, Alb: albumin, BUN: blood urea nitrogen, Cr: creatinine, Na: sodium, K: potassium, Cl: Chloride, Ca: calcium, P: phosphorus, Mg: magnesium, AST: aspartate transaminase, ALT: alanine aminotransferase, LDH: lactate dehydrogenase, ALP: alkaline phosphatase, T-bil: total bilirubin, CPK: creatine phosphokinase, AMY: amylase, CRP: C-reactive protein, $\mathrm{PaCO}_{2}$ : arterial partial pressure of carbon dioxide, $\mathrm{PaO}_{2}$ : arterial partial pressure of oxygen, $\mathrm{HCO}_{3}{ }^{-}$: bicarbonate, BE: base excess. Samples for blood gas analysis were collected under $2 \mathrm{~L} / \mathrm{min} \mathrm{O}_{2}$ administration.

topenia, and elevated creatinine phosphokinase was confirmed in addition to high concentrations of inflammatory markers (Table). Contrast-enhanced CT demonstrated massive emphysematous swelling from the pharynx to the mediastinum (Fig. 1A-D). In addition, sagittal CT showed anterior upper thoracic osteophytes at Th 3-4 with surrounding free air, indicative of esophageal perforation (Fig. 1E).

Upon returning from undergoing CT, he developed progressive dyspnea, and airway narrowing was identified by pharyngoscopy. He was taken to the operating room and intubated immediately. During the emergency operation, tracheostomy with cervical abscess drainage was performed. Subsequently, a thoracoscope was inserted into the mediastinum, and his bilateral pleural spaces were evaluated. Contaminated effusion was found in the posterior mediastinum around the entire esophagus, and mediastinal pus was drained. The posterior esophageal wall had strongly adhered to the vertebral bodies. A longitudinal esophageal tear was identified (Fig. 2A), and a sharp osteophyte (Fig. 2B) that had breached the esophageal serosa, extending inwards into the mucosa, and caused the perforation was identified by diligent observation (Fig. 3A and B). There were no gross abnormalities, such as malignancy or diverticulum at the perforation site; we therefore suspected that the perforation occurred following direct compression by the osteophyte.

Because the contamination was severe, primary recon- structive surgery was avoided, and thoracoscopic subtotal esophagectomy was performed instead. The offending osteophyte was smoothed using bone rongeurs. The posterior mediastinum and the cervical area were irrigated with saline and widely drained. Finally, a gastrostomy feeding tube was introduced, which completed the first surgery.

The patient was transported to the intensive-care unit because of hemodynamic instability. He was treated according to the global sepsis guidelines along with vasopressor therapy, and stress-dose corticosteroids were initiated as adjunctive therapy. Empiric broad-spectrum antibiotics (meropenem, 2 g/day) were administered, considering his renal function, and he recovered from the septic state and renal dysfunction within $96 \mathrm{~h}$. Because bacterial cultures of intraoperative samples yielded non-drug resistant bacteria (mainly Streptococcus constellatus and Streptococcus mitis), the antimicrobials were de-escalated to ampicillin-sulbactam (12 g/day). Follow-up radiological studies revealed residual abscesses concomitant with osteomyelitis, and additional drainage was required on the 14th day of hospitalization. Continued antibiotics were planned for four to six weeks for osteomyelitis. His general condition as well as laboratory values gradually improved, and he was transferred for rehabilitation on the 46th day of hospitalization. Elective esophageal reconstruction surgery was planned after his nutritional status improved. 

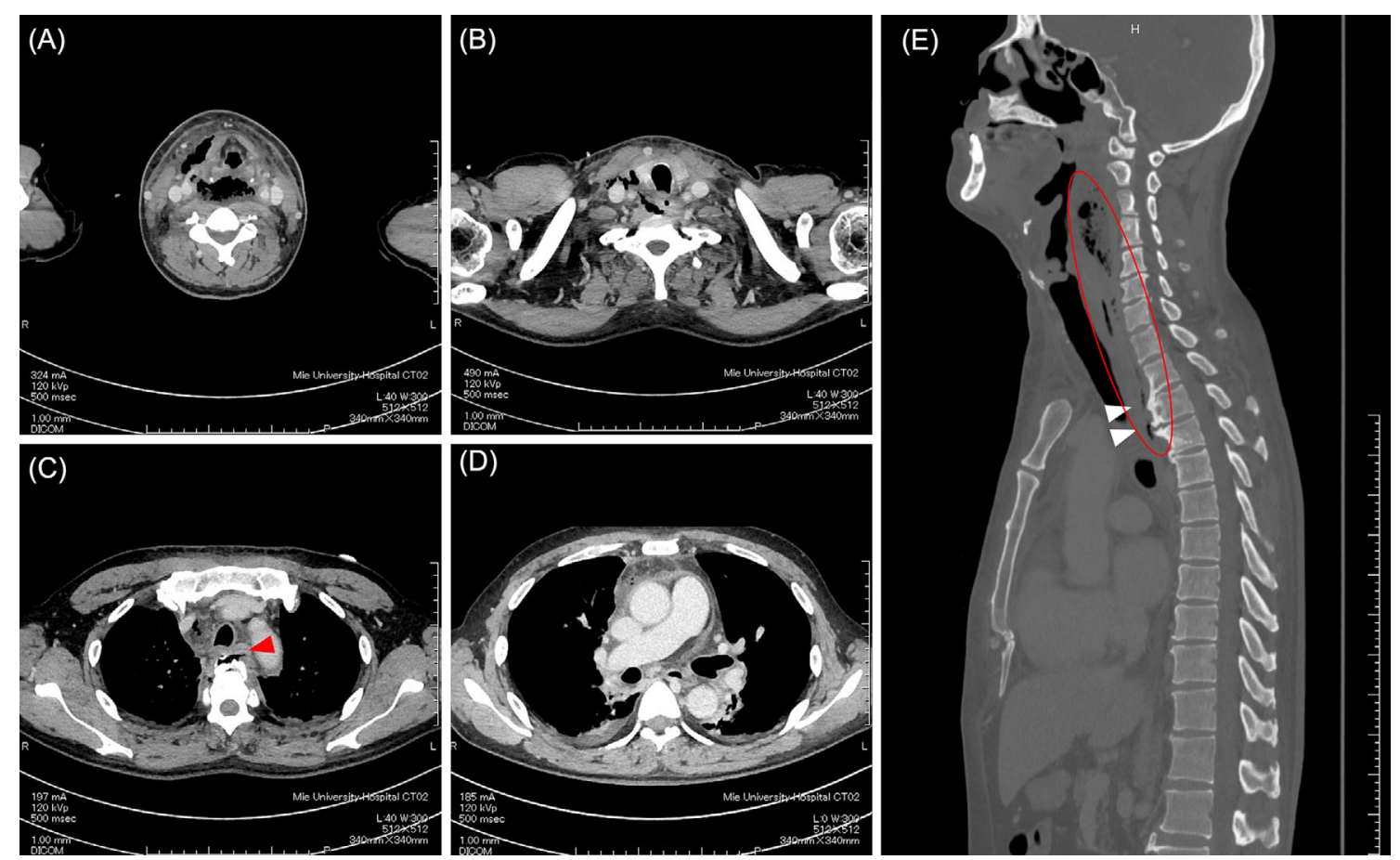

Figure 1. CT images at presentation to our hospital. (A-D) Contrast-enhanced chest CT of the trunk showing air in the mediastinal soft tissues extending to the retropharyngeal space, indicating abscess formation. (E) Reconstituted sagittal CT image showing Th 3-4 osteophytes and esophageal compression with air, indicating esophageal perforation.
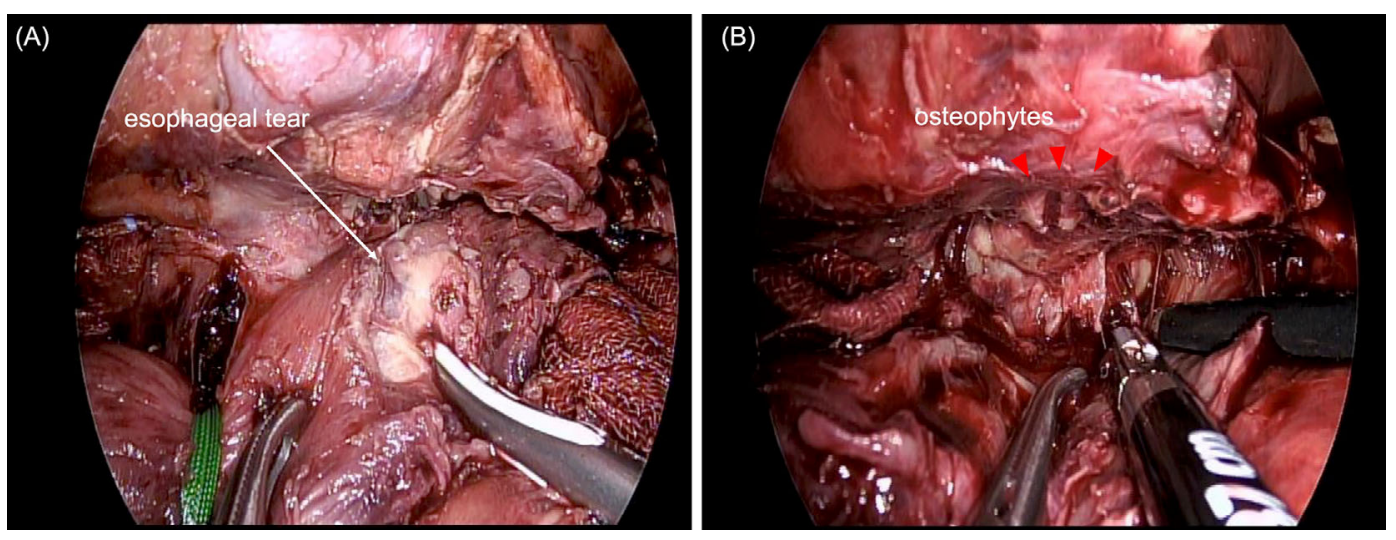

Figure 2. (A, B) Surgical findings. An esophageal tear (A: white arrow) and large, sharp osteophytes (B: red arrowheads) bulging from the vertebral bodies.
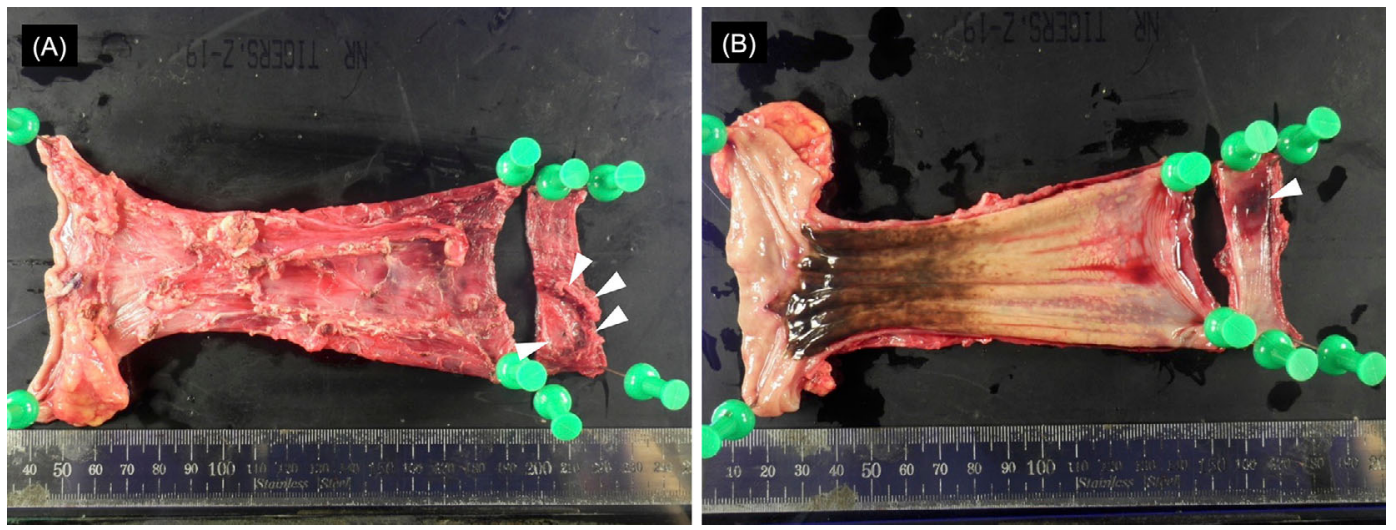

Figure 3. (C, D) The esophageal surgical specimen. An esophageal tear was found originating from the serosa (C: white arrowhead) and extending into the lumen (D: white arrowhead). 


\section{Discussion}

To our knowledge, this is the first case report of esophageal perforation with mediastinitis leading to retrograde cervical abscesses resulting from thoracic osteophytes following blunt trauma. This case highlights several important clinical insights: (i) thoracic osteophytes can cause esophageal perforation resulting from hyperextension in closed trauma injury; (ii) because esophageal perforation by osteophytes usually causes minimal injury, acutely, a prompt diagnosis is difficult; and (iii) a delayed diagnosis may contribute to the development of retrograde cervical abscesses.

Anterior osteoarthritic osteophytes are very common in older adult patients (3). Osteogenesis occurs because of repetitive mechanical factors involving the cartilage-periosteal attachment and capsule-ligament traction areas, which occurs predominantly in the upper thoracic and lower cervical spine (4). Although large thoracic osteophytes cause dysphagia, most remain asymptomatic despite their location and prevalence (3). Esophageal perforations are a relatively rare condition but are a difficult problem with a high mortality rate $(1,2)$. Iatrogenic causes have been implicated in up to $73 \%$ of esophageal perforations, and the majority occur with endoscopy (5). Although the relationship between spinal osteophytes and esophageal perforation is not well documented, some researchers have reported the need for careful attention to osteophytes when performing transesophageal echocardiography (TEE) (6-8). Direct pressure applied to the TEE probe against sharp vertebral osteophytes led to esophageal perforation in some cases. In thoracic lesions, the esophagus is vulnerable to extrinsic compression by anterior osteophytes, particularly at the tracheal bifurcation, as the anatomical space between these structures and the vertebral column is small $(8,9)$. Unlike "cervical" osteophytes $(2,3)$, esophageal perforation as a result of "thoracic" osteophytes is much rarer(1). In our case, hyperextension-flexion of the thoracic spine in blunt trauma caused by a fall resulted in extrinsic esophageal compression and esophageal perforation (2).

A delayed diagnosis of digestive tract perforation significantly influences the prognosis. In the present case, there was a 4-day delay between the injury and the first surgery. Because the inner cavity around the esophagus was small, and gut contents were absent, unlike in other parts of the gastrointestinal tract, microperforation by osteophytes was unclear acutely, based on the patient's initial symptoms. If not detected and treated early, these perforations may cause mediastinitis.

The prevalence of spinal injuries remains high in the elderly, and more than $75 \%$ of people over 65 years old have vertebral changes (3). Because esophageal perforation by osteophytes is rare but life-threatening, a high index of suspicion and sometimes repeat CT imaging is needed in elderly patients, especially those with a history of fall-related injury. Patient education regarding the delayed development of esophageal perforation is also important.

In the present case, mediastinitis was exacerbated by secretions and the patient's oral intake, progressing to retrograde cervical abscess formation. The standard treatment for such advanced cases has not yet been established; however; conservative treatment as well as simple drainage is considered insufficient, and an extensive and multidisciplinary surgical approach is often necessary. Whether or not primary surgical repair is feasible depends on the operative findings, but elective reconstructive surgery should be considered in highly contaminated cases. Whether or not osteophytes should be removed during the primary surgery is unclear, as doing so may increase the risk of developing osteomyelitis (2).

In conclusion, thoracic osteophytes are associated with a rare but important risk of esophageal perforation. The symptoms may be relatively mild acutely; patients may therefore visit internal medicine services several days after a related injury. Clinicians should be aware of this pathological condition and consider this diagnosis in elderly patients with a history of blunt trauma, especially a fall.

\section{The authors state that they have no Conflict of Interest (COI).}

\section{Acknowledgement}

The authors thank all of their colleagues in the Emergency and Critical Center, Mie University Hospital (Drs K Sasaki, K Hachiya, K Ikejiri, Y Akama, T Shinkai, A Ito-Masui, G Miyamura, E Kawamoto, K Yokoyama, and K Ishikura), Drs S Kaneda and A Ito (Department of Thoracic and Cardiovascular Surgery, Mie University Hospital), and Drs K Ueda, and K Chiyonobu (Department of Otorhinolaryngology, Head and Neck Surgery, Mie University Hospital) for their assistance. We thank Jane Charbonneau, DVM, from Edanz (https://jp.edanz.com/ac) for editing a draft of this manuscript.

\section{ICMJE authorship criteria}

KS was responsible for the organization and coordination of this case report. HY and RE contributed to the interpretation of the results. All authors were involved in the diagnosis, management, and/or care of the patient. All authors have read and approved the final manuscript.

Kei Suzuki and Haruna Yoshida contributed equally to this work.

\section{References}

1. Rathinam S, Makarawo T, Norton R, Collins FJ. Thoracic osteophytes: rare case of esophageal perforation. Dis Esophagus 23: E5-E8, 2010.

2. Henderson E, Echava V, Lalancette M, Langlois G. Esophageal perforation in closed neck trauma. Can J Surg 50: E5-E6, 2007.

3. Evans D, Luizza A, Zanders T, Jeanmonod R. Elderly man with dysphagia and esophageal perforation from an anterior cervical osteoarthritic osteophyte. Am J Emerg Med 33: 1711.e3-1711.e4, 2015.

4. Lencerf $\mathrm{P}$, Malard O. How to diagnose and treat symptomatic an- 
terior osteophytes? Eur Ann Otorhinolaryngol Head Neck Dis 127: 111-116, 2010.

5. Keiv J, Amendla M, Bouhaidar D, Sandhu B, Zhao X, Maher J. A management algorithm for esophageal perforation. Am J Surg 194: 103-106, 2007.

6. Bavalia N, Anis A, Benz M, Maldjian P, Bolanowski PJ, Saric M. Esophageal perforation, the most feared complication of TEE: early recognition by multimodality imaging. Echocardiography $\mathbf{2 8}$ : E56-E59, 2011

7. Chang K, Barghash M, Donnino R, Freedberg RS, Hagiwara M, Bennett G, et al. Extrinsic esophageal compression by cervical osteophytes in diffuse idiopathic skeletal hyperostosis: a contraindication to transesophageal echocardiography? Echocardiography 33:
314-316, 2016.

8. Royer O, Couture ÉJ, Nicodème F, Kalavrousiotis D, Maruyama TC, Denault A, et al. Esophageal perforation with transesophageal echocardiography in an elderly patient with prominent vertebral osteophytes: a case report and a review of the literature. CASE (Phila) 25: 331-336, 2020.

9. Klaassen Z, Tubbs RS, Apaydin N, Hage R, Jordan R, Loukas M. Vertebral spinal osteophytes. Anat Sci Int 86: 1-9, 2011.

The Internal Medicine is an Open Access journal distributed under the Creative Commons Attribution-NonCommercial-NoDerivatives 4.0 International License. To view the details of this license, please visit (https://creativecommons.org/licenses/ by-nc-nd/4.0/).

(C) The Japanese Society of Internal Medicine Intern Med Advance Publication 